\title{
Leishmania braziliensis
}

National Cancer Institute

\section{Source}

National Cancer Institute. Leishmania braziliensis. NCI Thesaurus. Code C123507.

A species of parasitic trypanosomatid protozoa in the family Trypanosomatidae. L. braziliensis is a New World pathogen. As well as causing localized cutaneous lesions in affected people, this species can also result in a destructive, progressive infection of mucosal tissue. 